\title{
MULTI-LEVEL OPTIMIZATION APPROACH TO MANAGING THE DEVELOPMENT OF HIERARCHICAL SOCIO-ECONOMIC SYSTEMS
}

\author{
Roman A. Zhukov \\ Financial University Under the Government of the Russian Federation (Tula Branch), Tula, Russian Federation
}

\begin{abstract}
The purpose of the article is to develop a multi-level optimization approach to the management of hierarchical socio-economic systems (HSES). The starting point of the approach is to build a model of the functioning of HSES at the regional level (meso-level), which includes a set of models that characterize the results of the functioning of elements and their aggregates that form subsystems - classes - within the framework of a formalized description of the object of research. For each element and subsystem (for the selected subsystems, its own model of functioning can be built) of the considered hierarchy level, particular and integral indicators of performance, efficiency and harmony, which serve as indicators of the set (normative, expected) functioning of HSES, are formed. If indicators deviate from standard values, an optimization procedure is performed, which consists in searching for such conditions (state and impact factors for each of the considered subsystems and their elements), in which indicator values will correspond to the standard (expected) values. In general, this problem is reduced to the problem of multi-criteria optimization, where the target function consists of a combination of private and aggregated production functions, the parameters of which are determined using the factor analysis of dependencies. Object and cybernetic models illustrating the multi-level optimization approach to the management of HSES are presented.

Key words: multi-level optimization approach, performance indicator, hierarchy, optimization, hierarchical socio-economic system, production function.
\end{abstract}

Citation. Zhukov R.A. Multi-Level Optimization Approach to Managing the Development of Hierarchical Socio-Economic Systems. Journal of Volgograd State University. Economics, 2020, vol. 22, no. 3, pp. 17-29. (in Russian). DOI: https://doi.org/10.15688/ek.jvolsu.2020.3.2

УДК 330.8:330.4:519.86

Дата поступления статьи: 06.09.2020

ББК 65 в 631

Дата принятия статьи: 28.09.2020

\section{МНОГОУРОВНЕВЫЙ ОПТИМИЗАЦИОННЫЙ ПОДХОД К УПРАВЛЕНИЮ РАЗВИТИЕМ ИЕРАРХИЧЕСКИХ СОЦИАЛЬНО-ЭКОНОМИЧЕСКИХ СИСТЕМ}

\section{Роман Александрович Жуков}

Тульский филиал Финансового университета при Правительстве РФ, г. Тула, Российская Федерация

\begin{abstract}
Аннотация. Целью статьи является разработка многоуровневого оптимизационного подхода к управлению иерархическими социально-экономическими системами (ИСЭС). Отправная точка подхода - построение модели функционирования ИСЭС на региональном уровне (мезоуровень), которая включает набор моделей, характеризующих результаты функционирования элементов и их совокупностей, образующих подсистемы - классы - в рамках формализованного описания объекта исследования. Для каждого элемента и подсистемы (для выделенных подсистем может быть построена собственная модель функционирования) рассматриваемого уровня иерархии формируются частные и интегральные показатели результативности, эффективности и гармоничности, которые служат индикаторами заданного (нормативного, ожидаемого) функционирования ИСЭС. При отклонении индикаторов от нормативных значений проводится процедура оптимизации, заключающаяся в поиске таких условий (факторов состояния и воздействия для каждых из () рассматриваемых подсистем и их элементов), при которых значения индикаторов будут соответствовать
\end{abstract}




\section{ЭКОНОМИЧЕСКАЯ ТЕОРИЯ}

нормативным (ожидаемым) значениям. В общем случае такая задача сводится к задаче многокритериальной оптимизации, где в состав целевой функции входят комбинации частных и агрегированных производственных функций, параметры которых определяются с помощью факторного анализа зависимостей. Представлены объектная и кибернетическая модели, иллюстрирующие многоуровневый оптимизационный подход к управлению ИСЭС.

Ключевые слова: многоуровневый оптимизационный подход, показатель результативности, иерархия, оптимизация, иерархическая социально-экономическая система, производственная функция.

Цитирование. Жуков Р. А. Многоуровневый оптимизационный подход к управлению развитием иерархических социально-экономических систем // Вестник Волгоградского государственного университета. Экономика. - 2020. - Т. 22, № 3. - C. 17-29. - DOI: https://doi.org/10.15688/ek.jvolsu.2020.3.2

\section{Введение}

Существующая система управления на различных уровнях (государство, округ, субъект Российской Федерации, муниципальное образование, хозяйствующий субъект) подразумевает необходимость согласования принимаемых управленческих решений, позволяющих обеспечить достижение как стратегических, так и тактических целей, направленных на устойчивое развитие РФ и повышение качества жизни населения. Сложность управления такой системой, которую будем называть иерархической социально-экономической системой (далее - ИСЭС), заключается в наличии множества межуровневых и внутриуровневых связей как между субъектами, так и между объектами управления, что требует планомерной трансформации традиционных методов управления и их интеграции с современными достижениями науки в области информатизации и математического моделирования. Неслучайно в подавляющем большинстве регионов РФ используются различные информационные системы, позволяющие упростить взаимодействие соответствующих органов с целью оптимизации процедур согласования решений различного уровня. Однако в качестве ключевого метода используется метод экспертных оценок, отодвигая на второй план разрабатываемый для этих целей математический аппарат и различные методы моделирования, последние из которых позволяют обосновать и ускорить процесс принятия решений на различных уровнях.

В этом аспекте актуальным представляется концептуальное и формализованное описание такого процесса, включающего характеристику ИСЭС, конструирование индикаторов ее оценки различного типа, а также использо- вание этапов анализа и синтеза управленческих решений в рамках новой теории экономических систем для обеспечения заданных (желаемых) результатов функционирования объекта исследования, представляющего собой иерархическую структуру.

Целью исследования является разработка многоуровневого оптимизационного подхода к управлению развитием ИСЭС.

\section{Состояние проблемы и обзор исследований}

Современные концептуальные и формализованные описания иерархических социально-экономических систем базируются на классических работах Т. Саати [Саати, 1993] и М. Мексаровича [Месарович и др., 1973], в которых введены такие понятия, как «иерархия», «уровень», «страта», «слой», и которые позволяют выделить иерархические системы среди остальных структур и формально их описать в терминах теории множеств. На базе таких структур решается ряд оптимизационных задач, связанных как с оптимизацией иерархических структур [Воронин и др., 2003], так и с принятием оптимальных решений для одного элемента или группы элементов уровня [Андрейчиков и др., 2015] на множестве вариантов по Парето [Ногин, 2002].

В рамках описания иерархических структур должна быть осуществлена классификация элементов, позволяющая объединить их в однотипные группы (классы). Примерами таких классификаций являются: классификация С.В. Белоусовой (общая классификация социально-экономических систем) [Белоусова, 2015]; пространственно-временная классификация Г.Б. Клейнера на базе новой теории экономических систем [Клейнер, 2016]; клас- 
сификация И.К. Ларионова по типу хозяйственного механизма [Ларионов, 2001] и др. При всем многообразии классификаций, по сути, такая процедура объединения элементов дает возможность «взглянуть» на одну и ту же сложную систему (один и тот же процесс или явление) с той или иной точки зрения, воспринимая совокупность элементов как некоторую подсистему, функционирующую, опять же с точки зрения исследователя, как единое целое. То есть многообразие классификаций определяется целями и взглядами автора, принимающего либо традиционную, либо собственную классификацию, последняя из которых должна быть понятна не только автору, но и другим исследователям. В этом смысле процедура согласования результатов исследований может быть решена за счет введения таблицы (матрицы) сопряженности, устанавливающей соответствие изучаемых признаков в различных классификациях.

В случае если объект исследования идентифицирован: описана его структура (концептуально или формализованно), проведена классификация, то изучение (в данном аспекте оценка функционирования ИСЭС) можно осуществлять через его признаковые описания, которые, в свою очередь, определяются выбором соответствующих индикаторов, что представляет определенную проблему для исследователя. Многообразие классификаций и целей проводимых исследований порождает множество как частных, так и интегральных (для совокупности элементов ИСЭС) индикаторов оценки. Среди таких индикаторов можно выделить, например: индикаторы устойчивого развития [Человек ..., 2018]; показатели качества жизни населения [Айвазян, 2012]; индикаторы, определяемые по системе национальных счетов [Зандер и др., 2015]; валовой региональный продукт (ВРП, наиболее часто используется в региональных исследованиях при оценке экономической составляющей ИСЭС, а также его модификация - ВРП на душу населения при конструировании индекса состоятельности) [Жуков, 2019г]; показатели оценки функционирования отдельных сфер производства товаров и услуг [Региональная экономика, 2012]; другие макроэкономические показатели [Арженовский и др., 2014; Вероятностная модель ...,
2016; Черницкий, 2014]. Наряду с традиционными показателями оценки функционирования ИСЭС, могут быть использованы частные и интегральные показатели результативности, эффективности и гармоничности, обоснование применения которых отражено, например, в [Жуков, 2019в].

Наличие индикаторов оценки позволяет сравнивать заданные (нормативные, ожидаемые) результаты функционирования ИСЭС с их фактическими значениями, обеспечение соответствия которых друг с другом является основной задачей управления на различных уровнях иерархии. Следовательно, для решения этой задачи (управление развитием иерархических социально-экономических систем) необходимо сформировать такие условия за счет воздействий со стороны субъекта управления, которые бы позволили достигнуть ожидаемых результатов - целей функционирования объекта исследования. Формализация задачи управления, причем наиболее оптимальным (эффективным) способом, предопределяет необходимость построения как моделей самой ИСЭС (например, с использованием классических производственных функций [Cobb et al., 1928; Solow, 1956; Corbo et al., 1979]), так и модели управления на разных уровнях иерархии. Становление системы взглядов на процесс управления сложными системами, обзор которых представлен в [Формирование ..., 2018], обусловливает необходимость использования как технико-экономического, так и традиционного (классический менеджмент) подхода к управлению ИСЭС в рамках общей теории систем и системного анализа [Bertalanffy, 1962].

\section{Этапы подхода}

Разработанный многоуровневый оптимизационный подход к управлению ИСЭС предполагает реализацию следующих этапов: 1) формализованное описание ИСЭС; 2) идентификация результатов функционирования ИСЭС на каждом из уровней иерархии, а также факторов состояния и воздействия (управляющих факторов), характеризующих конкретные условия деятельности объекта исследования; 3) построение моделей связи между результативными признаками элементов (классов, уровней) ИСЭС и условиями, по ко- 
торым определяются нормативные (ожидаемые) значения, являющиеся целью функционирования ИСЭС; 4) оценка показателей результативности, эффективности и гармоничности функционирования ИСЭС, однотипных для каждого из иерархических уровней; 5) отбор результативных признаков, не соответствующих нормативным значениям; 6) оптимизация факторных признаков - поиск таких значений, при которых рассматриваемые результативные признаки, характеризующие функционирование элементов, классов, уровней и ИСЭС в целом достигали бы (находились в допустимых пределах) своих нормативов, то есть цели функционирования были бы достигнуты. Заметим, что значения нормативов в случае их задания для нескольких временных периодов можно ассоциировать (связать) с заданной траекторией развития ИСЭС.

По сути, последний этап представляет собой поиск оптимального решения при управлении развитием ИСЭС, который должен быть включен в соответствующий контур с обратной связью на базе принципа управления по отклонению (компенсации) на каждом цикле принятия решения. Контур управления может быть представлен в виде кибернетической и процессной моделей.

\section{Формализация подхода}

Формализация соответствующего подхода может быть представлена следующим образом.

1. Описание ИСЭС. Каждый элемент $k_{p,(p-1), v_{p}, s_{q}} \in L_{p,(p-1), s_{q}}$ множества $H$ - иерархии $<H, R>, R$ - отношение нестрогого порядка, начиная со второго уровня, нумеруется четырьмя индексами: $p$ - номер уровня $L_{p} ;(p-1)-$ индекс элемента, которому подчинен рассматриваемый элемент; $v_{p}$ - номер этого элемента; $s_{q}$ - номер класса - подмножества $L_{p,(p-1), s_{q}}$, к которому отнесен элемент. Примером может служить иерархия - государство, округ, регион (субъект РФ), муниципальное образование (далее - МО), хозяйствующий субъект (предприятие). Классификация может быть осуществлена разделением всех элементов на крупные социальный и экономический классы, последний из которых может быть разделен по пространственно-временному [Клейнер, 2019], сек- торальному [Колесников, 2016] признакам или в традиционном варианте - по разделам Общероссийского классификатора видов экономической деятельности (ОКВЭД).

2. Идентификация признаков. Каждый из элементов ИСЭС уровня $L_{p}$ характеризуется 4 типами признаков (допустимо отождествлять объекты с их признаковыми описаниями): 1) результативный признак $y_{p,(p-1), v_{p}, s_{q}}(t)-$ peзультат функционирования элемента в период времени $t, t=1, \ldots, T, T$ - число периодов (фактические значения результативного признака); 2) факторы состояния $x_{p,(p-1), v_{p}, s_{q}, j}(t)$ - условия функционирования элемента, $j=1, \ldots, J, J-$ число факторов состояния; 3) факторы воздействия $z_{p,(p-1), v_{p}, s_{q}, u}(t)$, которые характеризуют управляющие воздействия на элемент, $u=1, \ldots, U, U-$ число факторов воздействия; 4) нормативный результативный признак $\hat{y}_{p,(p-1), v_{p}, s_{q}}(t)$ - нормативный (ожидаемый) peзультат функционирования элемента.

3. Связи. Связи между результативными признаками и факторами состояния и воздействия могут быть представлены посредством неслучайных функций $f_{p,(p-1)}$, которые будем называть производственными функциями (далее - ПФ):

$$
\begin{aligned}
& \hat{y}_{p,(p-1), v_{p}, s_{q}}(t)= \\
& =f_{p,(p-1)}\left(x_{p,(p-1), v_{p}, s_{q}, j}(t), z_{p,(p-1), v_{p}, s_{q}, u}(t)\right) .
\end{aligned}
$$

Параметры ПФ могут быть найдены с помощью факторного анализа зависимостей из эконометрических уравнений вида

$$
y_{p,(p-1), s_{q}}=\widehat{y}_{p,(p-1), s_{q}}+\varepsilon,
$$

где $\varepsilon$ - стохастическая случайная составляющая (в первом приближении предполагается нормальной случайной величиной).

Выражение (1) характеризует внутриуровневые связи элементов ИСЭС. Формализованное описание связей классов может быть представлено в виде агрегированной ПФ:

$$
\sqrt{\sum_{i_{1}=1}^{I} \sum_{i_{2}=1}^{I} \widehat{r}_{p, i_{1}, i_{2}, s_{q}} \cdot \hat{y}_{p, i_{1}, v_{p}, s_{q}}(t) \cdot \hat{y}_{p, i_{1}, v_{p}, s_{q}}(t)}
$$

где $\widehat{r}_{p, i_{1}, i_{2}, s_{q}}-$ значения парного коэффициента корреляции Пирсона между $i_{1}$-ми $\hat{y}_{p, i_{1}, s_{q}}$ и $i_{2}$-ми $\hat{y}_{p, i_{2}, s_{q}}$ переменными - признаковыми описаниями $\left(i_{1}, i_{2}=1, \ldots, I\right)$. 
Межуровневые связи определяются как суммы соответствующих фактических признаковых описаний элементов или классов [например, среднегодовая численность занятых в субъектах РФ и в округе (сумма занятых во всех субъектах РФ, входящих в состав округа)].

Модели в форме (1) и (3) строятся по статистическим данным для совокупности $v_{p}$-х элементов, принадлежащих одному элементу (соотношение (1)) или одному классу (соотношение (3)). Примером таких данных могут служить статистические данные для 17 регионов Центрального федерального округа за несколько периодов времени. Если строить модель из набора частных и агрегированных ПФ только по данным одного региона (например, Тульская область), то такая модель $\tilde{y}_{p,(p-1), v_{p}, s_{q}}(t)$ (обозначим такие модели знаком « », в том числе для обозначения и других индикаторов, которые вычисляются только по данным для одного $v_{p}$-го субъекта) будет характеризовать функционирование только исследуемого субъекта РФ и будет выступать уже не в качестве модели для расчета норматива, а в качестве модели для вычисления фактических значений результатов его функционирования с определенной степенью точности, заданной этой моделью. С этих же позиций построенная модель будет характеризовать ожидаемое (нормативное) значение для всех подсистем (MO или хозяйствующих субъектов), входящих в состав рассматриваемого региона (для этого достаточно подставить фактические значения факторов состояния и воздействия конкретного МО).

В этом и состоит одна из особенностей подхода, заключающаяся в построении двух типов моделей: первая - для совокупности однотипных подсистем, вторая - для одной подсистемы. При такой постановке важен выбор точки отсчета - на каком уровне строить базовые модели, а затем по ним определять функционирование элементов (классов) ИСЭС на других уровнях иерархии. Например, строить модели функционирования элементов (классов) для совокупности МО, входящих в состав области, а функционирование области идентифицировать как сумму соответствующих результатов и условий, полученных для муниципальных образований.
В рамках исследования предложено в качестве отправной точки выбрать региональный уровень - мезоуровень, который обладает некоторой общностью имеющихся объектов и процессов, что позволяет использовать ряд моделей в виде производственных функций для описания его функционирования, что согласуется и с идеями А.А. Богданова, которого по праву признают одним из основоположников системного подхода к познанию мира [Богданов, 1989]. Он полагал, что изучение природы вещей следует начинать со среднего - мезо - уровня и через этот уровень искать подходы к пониманию мега-, макро- и микроструктуры мира.

4. Оценка функционирования ИСЭС на разных уровнях иерархии может быть осуществлена с помощью частных и интегральных индикаторов результативности, эффективности и гармоничности, которые формируют систему показателей, позволяющих комплексно изучить объект исследования. Конструирование этих индикаторов подробно отражено в [Жуков, 2019б], и в рамках исследования они имеют опосредованное, хотя и немаловажное значение и выступают в качестве целевых индикаторов в предлагаемом многоуровневом подходе.

В общем виде частные и интегральные показатели результативности можно определить как

$$
\begin{gathered}
\xi_{p,(p-1), v_{p}, s_{q}}(t)=\frac{y_{p,(p-1), v_{p}, s_{q}}^{0}(t)}{\widehat{y}_{p,(p-1), v_{p}, s_{q}}^{0}(t)}, \\
\xi_{p, v_{p}, s_{q}}(t)=\frac{\sqrt{\sum_{i_{1}=1}^{I} \sum_{i_{2}=1}^{I} r_{p, i_{1}, i_{2}, s_{q}} \cdot y_{p, i_{1}, v_{p}, s_{q}}^{0}(t) \cdot y_{p, i_{2}, v_{p}, s_{q}}^{0}(t)}}{\sqrt{\sum_{i_{1}=1}^{I} \sum_{i_{2}=1}^{I} \hat{r}_{p, i_{1}, i_{2}, s_{q}} \cdot \hat{y}_{p, i_{1}, v_{p}, s_{q}}^{0}(t) \cdot \hat{y}_{p, i_{1}, v_{p}, s_{q}}^{0}(t)}},
\end{gathered}
$$

где индекс 0 характеризует, что значения приведены к шкале от 0 до 1 после проведения процедуры стандартизации (случайная величина будет иметь нулевое математическое ожидание и единичную дисперсию, такие случайные величины будем обозначать индексом «*»); $r_{p, i_{1}, i_{2}, s_{q}}-$ значения парного коэффициента корреляции Пирсона (аналогично (3)).

Если выражения (4) и (5) близки к единице, то можно говорить о соответствии функционирования ИСЭС ожидаемым (нормативным) результатам. 
Оценку сбалансированности, в смысле соответствия совокупности показателей результативности нормативным значениям, можно осуществить с помощью коэффициента гармоничности, который определяется по формуле

$$
H_{A p}(t)=1-\frac{\sigma\left(\xi_{p, i}(t)\right)}{M\left(\xi_{p, i}(t)\right)},
$$

где $M\left(\xi_{p, i}(t)\right)$ - математические ожидания; $\sigma\left(\xi_{p, i}(t)\right)$ среднеквадратические отклонения; $i=1, \ldots, Q, Q$-число интегральных показателей результативности, соответствующих числу классов.

Чем ближе $H_{A p}(t)$ к единице, тем более сбалансированно (гармонично) функционирование рассматриваемого объекта исследования.

Набор частных и интегральных показателей эффективности можно представить по формуле

$$
E f(t)=\frac{\Delta y(t)}{\Delta x(t)}=\frac{y(t) / y\left(t_{o}\right)}{x(t) / x\left(t_{0}\right)} .
$$

Здесь $y(t), y\left(t_{0}\right), x(t), x\left(t_{0}\right)$ - соответственно результат функционирования элемента (подсистемы) и его (ее) факторный признак текущего и базисного либо предыдущего периода. Тогда (6) определяет базисные и цепные эффективности. В зависимости от подстановки соответствующих индикаторов в числитель [частный или интегральный показатель результативности (соотношения (4) или (5)) или коэффициент гармоничности] и знаменатель [факторы состояния, воздействия или их комбинации, аналогичные (3)] можно получить систему частных и интегральных показателей эффективности функционирования, воздействия и управления.

5. В качестве критериев, на основании которых принимается решение о необходимости проведения оптимизации, могут выступать частные и интегральные показатели результативности, а также коэффициент гармоничности. Их нормативные значения принимаются равными единице и являются целевыми показателями функционирования ИСЭС:

$$
\bar{\xi}_{p,(p-1), v_{p}, s_{q}}(t)=\bar{\xi}_{p, v_{p}, s_{q}}(t)=\hat{H}_{A p}=1 .
$$

Сформируем следующие условия:

$$
\begin{aligned}
& \xi_{p,(p-1), v_{p}, s_{q}}(t) \in \bar{\xi}_{p,(p-1), v_{p}, s_{q}}(t) \pm \delta_{p,(p-1), v_{p}, s_{q}}(t) \\
& \xi_{p, v_{p}, s_{q}}(t) \in \bar{\xi}_{p, v_{p}, s_{q}}(t) \pm \delta_{p v_{p}, s_{q}}(t) \\
& H_{A p}(t) \in \widehat{H}_{A p}(t)-\delta_{A p}(t)
\end{aligned}
$$

где $\delta_{p,(p-1), v_{p}, s_{q}}(t), \delta_{p v_{p}, s_{q}}(t)$ и $\delta_{A p}(t)$ - соответствующие доверительные интервалы, которые можно задать априорно ( $1 \%, 5$ \%, 10 \% и т. п.) или определить по формуле

$$
\delta=t_{1-\alpha ; n-p-1} \cdot s \cdot \sqrt{1+A_{0}^{T} \cdot\left(A \cdot A^{T}\right)^{-1} A_{0}},
$$

где $t_{1-\alpha ; n-p-1}-$ коэффициент, отвечающий за доверительную вероятность траектории; $\alpha$ - уровень значимости; $n$ - число наблюдений; $p$ - число параметров модели; $s$ - среднеквадратическая ошибка модели; $A$ - матрица известных значений факторов состояния и воздействия; $A^{0}$ - вектор ожидаемых значений факторов состояния и воздействия.

Если условия (9) выполняются, то функционирование ИСЭС признается удовлетворительным. В противном случае необходима оптимизация.

6. Процедура оптимизации. Пусть цель на уровне иерархии $L_{p}, p=1, \ldots, h$ определяется некоторым индикатором $\xi_{p}$, который является элементом множества признаковых описаний $D_{g}$ объекта (элемента) $k_{p}$ (в данном случае индикатор отображает результат функционирования ИСЭС на $L_{p}$ ). Если элемент множества $k_{p}$ есть объект, состоящий из совокупности объектов (то есть сам представляет собой некоторое подмножество) $k_{p, i, q} \in L_{p}, i=1, \ldots, N_{p}\left(N_{p}\right.$ - количество элементов множества на заданном уровне иерархии), сопоставленных с элементами множества наименований (номеров) классов $s_{q} \in S$, то цель для $\forall k_{p, i, q}$ можно сопоставить с индикатором $\xi_{k, p, q}$, который является элементом множества признаковых описаний $D_{\xi_{k, p, q}}$ объекта $k_{p, i, q} \in L_{p, q} \subset L_{p}, L_{p, q} \rightarrow s_{q} \in S$.

Введем функцию $\omega_{p}: \xi_{p, q} \rightarrow \xi_{p}, q=1, \ldots, s$ $(s-$ число классов), которая будет иметь смысл агрегирования целей $k_{p, i, q}$ на заданном уровне иерархии $p$. Тогда $\xi_{p}=\omega_{p}\left(\xi_{p, q}\right)$ будет определять цель на $L_{p}$.

В простейшем случае

$$
\xi_{p}=\sum_{q=1}^{s} \omega_{p, q} \cdot \xi_{p, q}
$$

где $\sum_{q=1}^{s} \omega_{p, q}=1$ и $\omega_{p, q}-$ весовая функция, отражающая приоритет относительно цели $\xi_{p, q}$. 
Такое формальное описание позволяет идентифицировать цели моделирования развития ИСЭС для каждого уровня иерархии.

Далее, поставим следующую общую задачу. Требуется найти такие значения приращений факторов состояния $\Delta x_{p,(p-1), v_{p}, s_{q}, j}(t)$ и факторов воздействия $\Delta z_{p,(p-1), v_{p}, s_{q}, u}(t)$, для которых целевая функция (11) и ее составляющие стремятся к единице.

Эта задача может быть сведена к одному из 7 типов задач многокритериальной оптимизации, рассмотренной в [Жуков, 2019а], с тем отличием, что вместо фактических значений результативного признака $y_{p,(p-1), v_{p}, s_{q}}(t)$ берется значение $\tilde{y}_{p,(p-1), v_{p}, s_{q}}(t)$, вычисленное по данным только для $v_{p}$-го элемента или класса.

Для устранения необходимости придания некоторых значений весовым коэффициентам в (11) с помощью экспертных оценок можно воспользоваться расширением метода, основанным на методологии анализа среды функционирования (АСФ) [Кривоножко и др., 2010], и метода Лагранжа, сформировав целевую функцию в следующем виде (при добавлении в соотношение коэффициента гармоничности в числитель добавляется соответствующее слагаемое с коэффициентом):

$$
\begin{aligned}
& F_{v_{p}}(t)= \\
& =\frac{\mu \cdot\left(\widetilde{y}_{p, v_{p}, s_{q}}^{0}(t)-\hat{y}_{p, v_{p}, s_{q}}^{0}(t)\right)+\sum_{i=1}^{(p-1)} \mu_{i} \cdot\left(\widetilde{y}_{p, i, v_{p}, s_{q}}^{0}(t)-\tilde{y}_{p, i, v_{p}, s_{q}}^{0}(t)\right)}{\sum_{i=1}^{(p-1)} \sum_{j=1}^{J} \omega_{i, j} \cdot\left|\Delta x_{p, i, v_{p}, s_{q}, j}^{0}(t)\right|+\sum_{i=1}^{(p-1)} \sum_{u=1}^{U} \eta_{i, u} \cdot\left|\Delta z_{p, i, v_{p}, s_{q}, u}^{0}(t)\right|} \rightarrow(12)
\end{aligned}
$$

где $\mu, \mu_{i} \omega_{i, j}, \eta_{i, u}$ - соответствующие весовые коэффициенты обобщенного и частных показателей результативности, а также факторов состояния и воздействия. В данном соотношении приращения факторных признаков будем считать нормализованными, то есть приведенными к интервалу от 0 до 1. Числитель представляет собой сумму разностей модельного и нормативного значений для обобщенного и частных показателей результативности $v_{p}$-го элемента (класса, подсистемы), которые должны стремиться к максимуму. Знаменатель по сути есть требование минимизации затрат на реализацию мероприятий, направленных на достижение нормативных значений функционирования рассматриваемой сложной системы.

Аналогично [Жуков, 2019а], воспользовавшись методом Лагранжа и преобразованием
Чарнеса и Купера для задач дробно-линейного программирования [Polyhedral ..., , p. 73], задачу можно свести к следующему виду:

$$
F(t)=\vartheta_{\mu}+\sum_{i=1}^{(p-1)} \vartheta_{i} \rightarrow \max ,
$$

где $\vartheta_{\mu}, \vartheta_{i}-$ преобразованные от $x_{p, i, v_{p}, s_{q}, j}^{0}(t)$ и $z_{p, i, v_{p}, s_{q}, u}^{0}(t)$ переменные с системой ограничений:

$$
\begin{gathered}
\sum_{i=1}^{m} \sum_{j=1}^{J} v_{i, j}+\sum_{i=1}^{m} \sum_{u=1}^{U} v_{i, u}=1, \\
g_{l}\left(v_{i, j}, v_{i, u}\right)=0 .
\end{gathered}
$$

В простейшем случае можно оптимизировать частные показатели результативности по отдельности, то есть решать задачи линейного (если производственные функции линейны) и нелинейного программирования с ограничениями. В качестве инструментальных средств можно использовать программный комплекс «ЭФРА» [Программа ...] либо среду разработки Mathlab [Среда ...].

После того как формализован алгоритм поиска оптимального решения, строится контур управления в соответствии с предлагаемым многоуровневым оптимизационным подходом в виде кибернетической и процессной моделей.

\section{Кибернетическая и процессная модели управления ИСЭС на основе многоуровневого оптимизационного подхода}

В основе многоуровневого оптимизационного подхода лежит одноуровневый подход, предложенный в [Жуков, 2019б], отличающийся тем, что в контур управления вводится дополнительная модель, которая описывает функционирование конкретного $v_{p}$-го элемента (класса, подсистемы) и строится по данным только для этого объекта исследования.

Для наглядности обозначим модели, которые строятся по данным для совокупности объектов, подчиненных одному элементу (классу) более высокого уровня $(p-1)$, как ФО-модели (регионы федерального округа), а модели, что построены только по данным для $v_{p}$-го элемента (класса), как Р-модели (модели функционирования региона (области, субъекта РФ)). 


\section{ЭКОНОМИЧЕСКАЯ ТЕОРИЯ}

Кибернетическая модель представлена на рисунке 1.

Результаты функционирования ИСЭС разделяются на три направления: 1) объем продукции и услуг, который поставляется внешней среде $\left\{y_{i, \text { ext }}(t)\right\}$ (где $i-$ индекс продукции или услуг; ext - external; \{\} - вектор, компонентами которого являются $\left.y_{i, \mathrm{ext}}(t)\right)$; 2) $\left\{y_{i, \text { int }}(t)\right\}$ (где int - internal) - результаты, идущие на внутреннее потребление и накопление; 3) информация о результатах функционирования ИСЭС, ее состоянии и характере воздействия в рассматриваемый период. Сбор информации осуществляется через систему мониторинга. Результаты мониторинга сравниваются с данными, полученными по ФО-модели, и попадают в блок анализатора, который вычисляет целевые индикаторы: показатели результативности, эффективности и гармоничности, которые вычисляются с использованием результатов оценки по Р-модели и ФО-модели [данные подставляются соответственно в числитель и знаменатель соотношений (4) и (5)]. На данном этапе субъект управления осуществляет корректировку Р-модели с учетом вновь появившихся данных. В блоке формирования управляющих воздействий осуществляется процедура оптимизации в соответствии с целями субъекта управления, имеющимися расхождениями между результатами оценок по Р- и ФО-моделям (этапы 5 и 6 многоуровневого оптимизационного подхода), результатом реализации которого становятся рекомендации в части изменения факторов состояния и воздействия с учетом ограничений на внутренние и внешние ресурсы, позволяющие достигнуть нормативных (ожидаемых) значений результатов функционирования ИСЭС. Эти рекомендации используются для организации деятельности ИСЭС в следующем цикле.

Процессная модель в нотации IDEFX демонстрирует связь этапов цикла управления, используемых ресурсов и информации, обеспечивающих реализацию каждого из этапов (см. рис. 2).

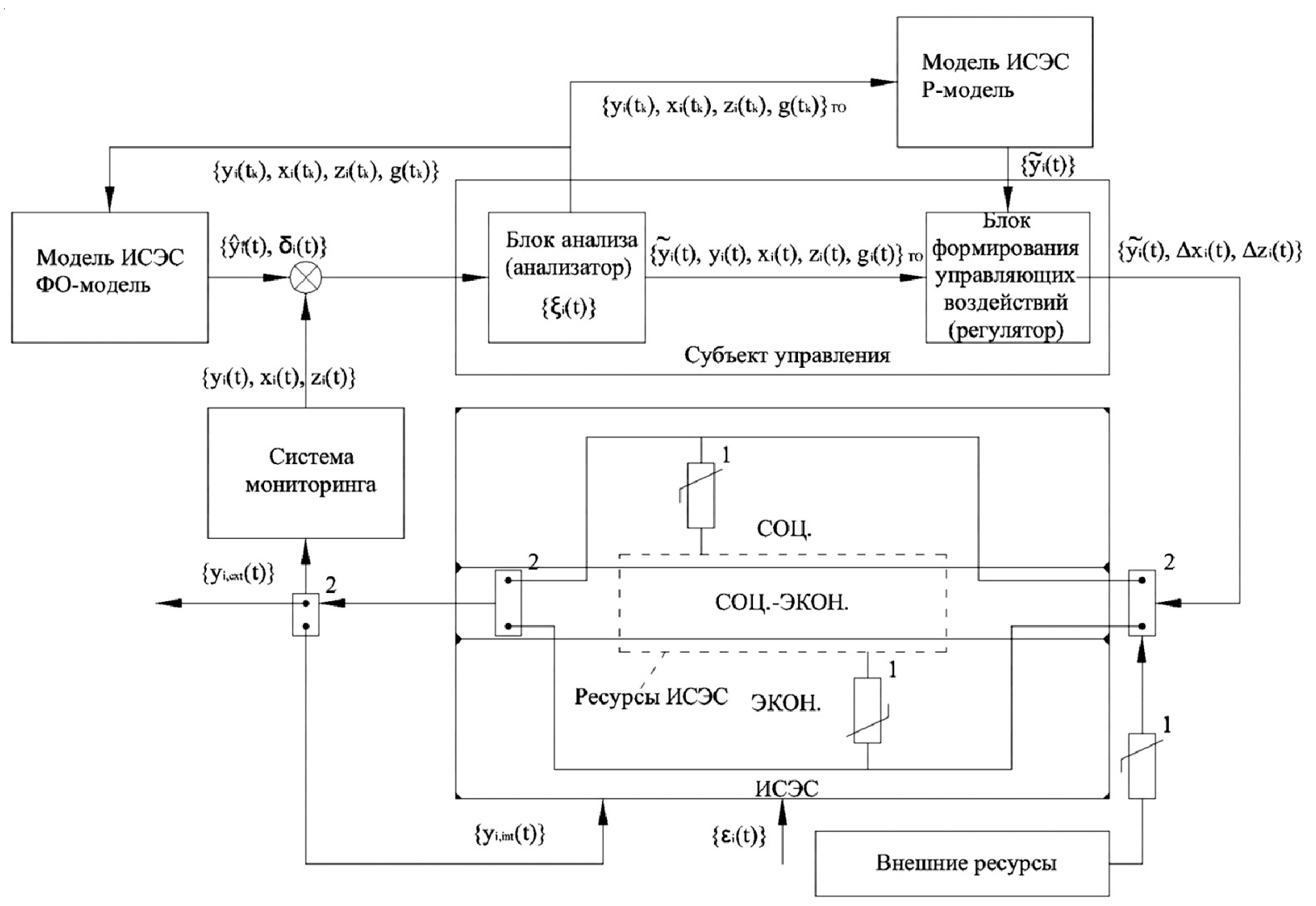

Рис. 1. Кибернетическая модель управления состоянием и развитием ИСЭС:

$$
1 \text { - ограничитель; } 2 \text { - разделитель }
$$

Примечание. Составлено автором. 


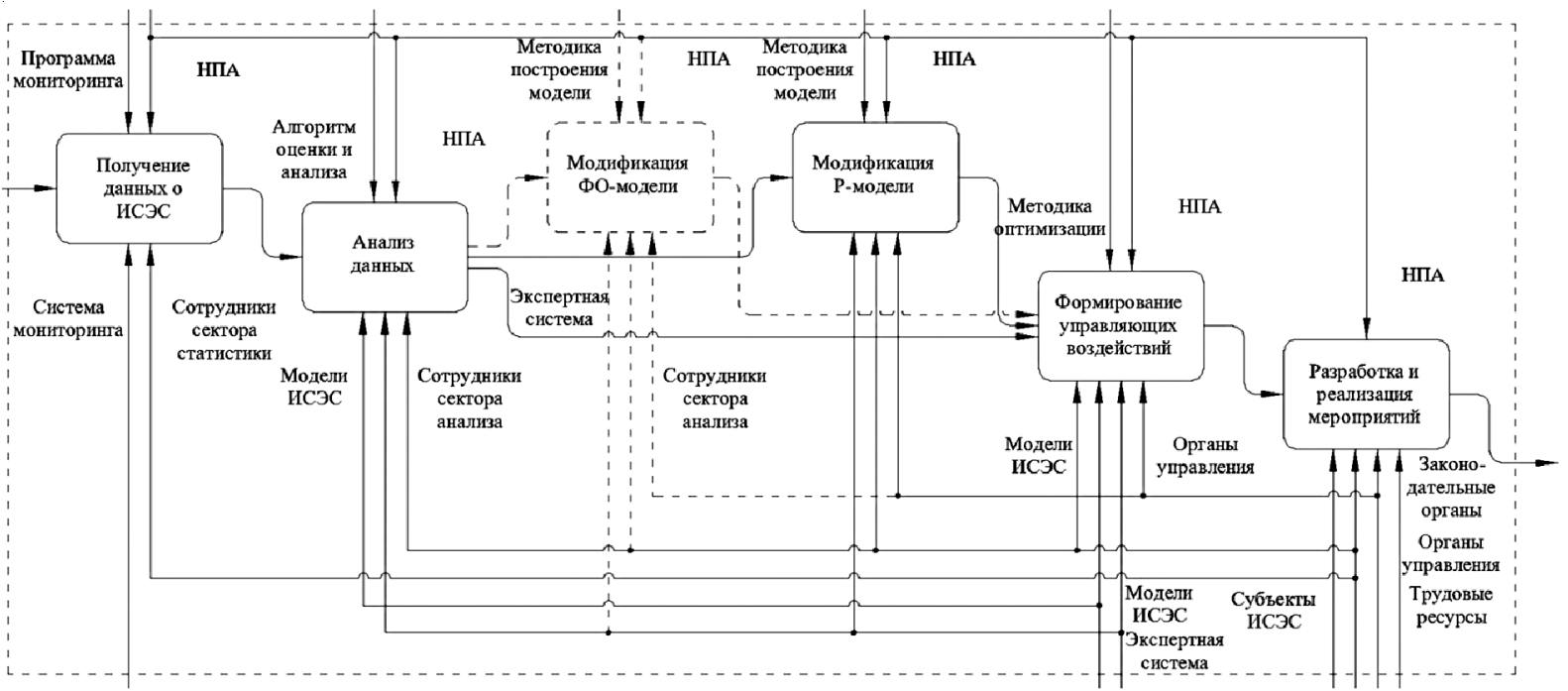

Рис. 2. Процессная модель управления состоянием и развитием ИСЭС

Примечание. Составлено автором.

Отличительной чертой процессной модели будет необходимая модификация Р-моделей на каждом цикле управления с учетом вновь появившихся данных.

Особенность данного подхода заключается в использовании двух типов моделей. Модели, относящиеся к первому типу, применяются для формирования нормативов и траектории развития (устойчивого развития), а модели, принадлежащие второму типу, - для поиска оптимальных значений факторов состояния и воздействия. Корректировка ФО-моделей (на рисунке 2 обозначено пунктирной линией) осуществляется не в каждом цикле, а в случае принятия решения об изменении нормативных (ожидаемых, плановых) показателей (целевых индикаторов), которые регламентируются, например, программой социальноэкономического развития субъекта РФ. Для повышения качества моделей и идентификации неучтенных факторов необходимо увеличить объем выборки, в том числе за счет разделения региона (области) на муниципальные образования и сбора данных по ним.

Однако, по крайней мере, на данном этапе это представляется серьезной проблемой, связанной с организацией статистических исследований, а также с раскрытием информации, определяемой федеральными законами Российской Федерации «Об информации, информационных технологиях и о защите информации» [Федеральный закон РФ от 27.07.2006 ...], «О го- сударственной тайне» [Федеральный закон РФ от 21.07.1993 ...] и «О коммерческой тайне» [Федеральный закон РФ от 29.07.2004 ...]. Именно поэтому в рамках исследования Рмодель строится по данным только для региона, а не по данным для совокупности МО, входящих в его состав.

Аналогичные модели управления будут характерны и для других уровней иерархии.

\section{Заключение}

В исследовании представлен многоуровневый оптимизационный подход к управлению развитием иерархических социально-экономических систем, заключающийся в использовании математического аппарата при принятии управленческих решений и позволяющий снизить влияние субъективизма со стороны лиц, принимающих решения. Он включает в себя: 1) концептуальное и формализованное описание объекта исследования (ИСЭС); 2) набор моделей 1-го типа, представленных в виде частных и агрегированных ПФ, которые используются для формирования нормативов - целевых значений индикаторов для элементов и подсистем, подчиненных только одному из элементов (подсистем) более высокого уровня; 3) набор моделей 2-го типа, строящихся только по статистическим данным одного элемента (подсистемы) заданного уровня ИСЭС, которые используются при 
проведении процедуры оптимизации - поиска таких факторов состояния и воздействия, при которых бы рассматриваемые элементы (подсистемы - классы, уровни, ИСЭС в целом) смогли достичь заданных (нормативных, ожидаемых) целевых значений индикаторов.

В рамках исследования построены кибернетическая и процессная модели управления развитием ИСЭС, являющиеся одними из составляющих предложенного подхода и позволяющие визуализировать и в дальнейшем реализовать алгоритм управления с помощью некоторых инструментальных средств поддержки принятия решений. В настоящее время такая работа проводится за счет расширения возможностей авторской экспертной системы «ЭФРА».

\section{СПИСОК ЛИТЕРАТУРЫ}

Айвазян, С. А. Анализ качества и образа жизни населения / С. А. Айвазян. - М. : Наука, 2012. $432 \mathrm{c}$.

Анализ методов принятия решений при разработке сложных технических систем / С.С. Семенов [и др.] // XII Всероссийское совещание по проблемам ВСПУ-2014, 16-19 июля 2014 г. - М. : Ин-т проблем управления им. В.А. Трапезникова РАН. - С. 8101-8123.

Андрейчиков, А. В. Системный анализ и синтез стратегических решений в инноватике / А. В. Андрейчиков, О. Н. Андрейчикова. - М. : Ленанд, 2015. $-306 \mathrm{c}$.

Арженовский, И. В. Региональная экономика : учеб. пособие / И. В. Арженовский, М. Кий. Н. Новгород : ННГАСУ, 2014. - 208 с. - Электрон. текстовые дан. - Режим доступа: http:// biblioclub.ru/index.php?page $=$ book\&id $=427578$ (дата обращения: 26.03.2020). - Загл. с экрана.

Белоусова, С. В. Система управления общественным сектором в свете теорий управления социально-экономическими системами / С. В. Белоусова // Вопросы управления. - 2015. - № 6 (37). C. 135-146.

Богданов, А. А. Тектология : Всеобщая организационная наука. В 2 кн. Кн. 2. / А. А. Богданов. - М. : Экономика, 1989. - 351 с.

Вероятностная модель прогнозирования регионального развития производительных сил / М. Р. Сафиуллин [и др.] // Казанский экономический вестник. -2016. -№4 (24). - С. 94-99.

Воронин, А. А. Оптимальные иерархические структуры / А. А. Воронин, С. П. Мишин. - М. : ИПУ РАН, 2003. -214 c.
Жуков, Р. А. Некоторые задачи оптимизации управления социо-эколого-экономическими системами / Р. А. Жуков // Чебышевский сборник. 2019a. - T. 20, № 1 (69). - C. 370-388.

Жуков, Р. А. Одноуровневое управление социальноэкономическими системами на основе производственной функции / Р. А. Жуков // Актуальные проблемы экономики и права. - 2019б. T. 19, № 2. - С. 1162-1173.

Жуков, Р. А. Оценка эффективности функционирования социально-экономических систем на основе производственных функций: новый подход / Р. А. Жуков // Вестник Волгоградского государственного университета. Серия 3 , Экономика. - 2019в. - Т. 21, № 3. - С. 71-82. DOI: https://doi.org/10.15688/ek.jvolsu.2019.3.7.

Жуков, Р. А. Социо-эколого-экономические системы: теория и практика : монография / Р. А. Жуков. М. : ИНФРА-М, 2019. - 186 с.

Зандер, Е. В. Региональное управление и территориальное планирование : учеб. пособие/Е. В. Зандер, Е. В. Лобкова, Т. А. Смирнова. - Красноярск : Сиб. федер. ун-т, 2015. - 282 с.

Клейнер, Г. Б. Системная сбалансированность экономики России. Региональный разрез / Г. Б. Клейнер, М. А. Рыбачук // Экономика региона. 2019. - Т. 15, № 2. - С. 309-323.

Клейнер, Г. Б. Экономика. Моделирование. Математика : избр. тр. / Г. Б. Клейнер. - М. : ЦЭМИ PAH, 2016. $-856 \mathrm{c}$.

Колесников, Н. Г. Структурные изменения экономики Северо-Запада России: пространственный аспект / Н. Г. Колесников, О.В.Толстогузов // Балтийский регион. - 2016. - Т. 8, № 2.С. $30-47$.

Кривоножко, В. Е. Анализ деятельности сложных социально-экономических систем / В. Е. Кривоножко, А. В. Лычев. - М. : Макс Пресс, 2010. $-208 \mathrm{c}$.

Ларионов, И. К. Мезоэкономика : учеб. пособие / И. К. Ларионов. - М. : Дашков и К , 2001. $444 \mathrm{c}$.

Месарович, М. Теория иерархических многоуровневых систем / М. Месарович, Д. Мако, И. Такахара. - М. : Мир, 1973. - 344 с.

Ногин, В. Д . Принятие решений в многокритериальной среде: количественный подход / В. Д. Ногин. - М. : ФИЗМАТЛИТ, 2002. - 144 с.

Программа для ЭВМ «Программный комплекс для оценки функционирования сложных систем и принятия решений “ЭФРА” (“EFRA”)». Свидетельство Федеральной службы по интеллектуальной собственности, патентам и торговым знакам об официальной регистрации программы для ЭВМ № 220614151 от 26.03.2020. 
Региональная экономика : учебник / Т. Г. Морозова [и др.]. - М. : ЮНИТИ-ДАНА, 2012. - 527 с.

Саати, Т. Принятие решений: метод анализа иерархий / Т. Саати. - М. : Радио и связь, 1993. - 278 с.

Среда разработки Mathlab. - Электрон. текстовые дан. - Режим доступа: https://www.mathworks. com/products/matlab.html (дата обращения: 04.05.2020). - Загл. с экрана.

Федеральный закон Российской Федерации от 21.07.1993 № 5485-1 «О государственной тайне». - Доступ из справ.-правовой системы «КонсультантПлюс».

Федеральный закон Российской Федерации от 29.07.2004 № 98-Ф3 «О коммерческой тайне». - Доступ из справ.-правовой системы «КонсультантПлюс».

Федеральный закон Российской Федерации от 27.07.2006 № 149-Ф3 «Об информации, информационных технологиях и о защите информации». - Доступ из справ.-правовой системы «КонсультантПлюс».

Формирование системы взглядов на процесс управления устойчивым развитием региона / Р. А. Жуков [и др.] // Научные исследования и разработки. Экономика. - 2018. - Т. 6, № 6. - С. 48-53.

Человек и инновации : Доклад о человеческом развитии в Российской Федерации за 2018 год / под ред. С. Н. Бобылева, Л. М. Григорьева. - М. : Аналит. центр приПравительствеРФ, 2018. - 172 с.

Черницкий, С. В. Комплексная методика оценки экономического потенциала регионов / С. В. Черницкий // Историческая и социально-образовательная мысль. - 2014. - № 5 (27). - С. 208-213.

Bertalanffy, L. General System Theory - A Critical Review / L. Bertalanffy // General Systems. 1962. - Vol. 7. - P. 1-20.

Cobb, C. W. A Theory of Production / C. W. Cobb, P. H. Douglas // American Economic Review. 1928. - № 18. - P. 139-165.

Corbo, V. The Translog Production Function. Some Evidence from Establishment Data / V. Corbo, P. Meller // Journal of Econometrics. - 1979. № 10. - P. 193-199.

Polyhedral Cone-Ratio DEA Models with an Illustrative Application to Large Commercial Banks / A. Charnes [et al.] // Journal of Econometrics. - 1990. - Vol. 46, № 1-2. - P. 73-91.

Solow, R. M. AContribution to the Theory of Economic Growth / R. M. Solow // The Quarterly Journal of Economics. - 1956. - Vol. 70, № 1. - P. 65-94.

\section{REFERENCES}

Aivazian S.A. Analiz kachestva i obraza zhizni naseleniya [Analysis of the Quality and Lifestyle of the Population]. Moscow, Nauka Publ., 2012. $432 \mathrm{p}$.

Semenov S.S., PoltavskijA.V., Maklakov V.V., KryanevA.V. Analiz metodov prinyatiya resheniy pri razrabotke slozhnykh tekhnicheskikh system [Analysis of Decision-Making Methods in the Development of Complex Technical Systems]. XII Vserossiyskoe soveshchanie po problemam VSPU-2014, 16-19 iyulya 2014 g. [XII AllRussian Meeting on the Problems of VSPU-2014, July 16-19, 2014]. Moscow, Institut problem upravleniya im. V.A. Trapeznikova RAN, pp. 8101-8123.

Andreichikov A.V., Andreichikova O.N. Sistemnyy analiz $i$ sintez strategicheskikh resheniy $v$ innovatike [System Analysis and Synthesis of Strategic Decisions in Innovation]. Moscow, Lenand Publ., 2015. 306 p.

Arzhenovskij I.V., Kiy M. Regionalnaya ekonomika: ucheb. posobie [Regional Economics: Study Guide]. Nizhny Novgorod, NNGASU, 2014.208 p. URL: http://biblioclub.ru/index.php?page= book\&id=427578 (accessed 26 March 2020).

Belousova S.V. Sistema upravleniya obshchestvennym sektorom v svete teoriy upravleniya sotsialnoekonomicheskimi sistemami [Public Sector Management System in the Light of the Theories of Socio-Economic Systems]. Voprosy upravleniya [Management Issues], 2015, no. 6(37), pp. 135-146.

Bogdanov A.A. Tektologiya: Vseobshchaya organizatsionnaya nauka. V 2 kn. Kn. 2. [Tectology: General Organizational Science. In 2 Books. Book 2]. Moscow, Ekonomika Publ., $1989.351 \mathrm{p}$.

Safiullin M.R., Elshin L.A., Prygunova M.I., GalyavovA.A. Veroyatnostnaya model prognozirovaniya regionalnogo razvitiya proizvoditelnykh sil [A Probabilistic Model for the Forecast of Regional Development of Productive Forces]. Kazanskiy Ekonomicheskiy Vestnik, 2016, no. 4 (24), pp. 94-99.

Voronin A.A., Mishin S.P. Optimalnye ierarkhicheskie struktury [Optimal Hierarchical Structures]. Moscow, IPU RAN, 2003. 214 p.

Zhukov R.A. Nekotorye zadachi optimizatsii upravleniya sotsio-ekologo-ekonomicheskimi sistemami [Some Optimization Problems of the Management of Socio-Ecological-Economic Systems]. Chebyshevskiy sbornik, 2019a, vol. 20, no. 1, pp. 370-388.

Zhukov R.A. Odnourovnevoe upravlenie sosialnoekonomicheskimi sistemami na osnove proizvodstvennoy funktsii [Single-Level Management of Social-Economic Systems Based on Production Function]. Aktualnye problemy 
ekonomiki i prava [Actual Problems of Economics and Law], 2019b, vol. 19, no. 2, pp. 1162-1173.

Zhukov R.A. Otsenka effektivnosti funktsionirovaniya socialno-ekonomicheskikh sistem na osnove proizvodstvennykh funktsiy: novyy podkhod [Efficiency Evaluation of Functioning of SocioEconomic Systems Based on Production Functions: New Approach]. Vestnik Volgogradskogo gosudarstvennogo universiteta. Seriya 3. Ekonomika [Journal of Volgograd State University. Economics], 2019v, vol. 21, no. 3, pp. 71-82. DOI: https://doi.org/ 10.15688/ek.jvolsu.2019.3.7.

Zhukov R.A. Sotsio-ekologo-ekonomicheskie sistemy: teoriya i praktika: monografiya [Socio-Ecological and Economic Systems: Theory and Practice. Monograph]. Moscow, INFRA-M Publ., 2019. 186 p.

Zander E.V. Regionalnoe upravlenie i territorialnoe planirovanie: ucheb. posobie [Regional Governance and Spatial Planning. Study Guide]. Krasnoyarsk, Sibirskiy federalnyy universitet, 2015. 282 p.

Kleiner G.B., Rybachuk M.A. Sistemnaya sbalansirovannost ekonomiki Rossii. Regionalnyy razrez [System Balance of the Russian Economy: Regional Perspective]. Ekonomika Regiona [Economy of Region], 2019, vol. 15, no. 2, pp. 309-323.

Kleiner G.B. Ekonomika. Modelirovanie. Matematika: izbr. tr. [Economy. Modeling. Mathematics. Selected Works]. Moscow, TsEMI RAN, 2016. $856 \mathrm{p}$.

Kolesnikov N.G., Tolstoguzov O.V. Strukturnye izmeneniya ekonomiki Severo-Zapada Rossii: prostranstvennyy aspekt [Structural Changes in the Economy of the Russian Northwest: Spatial Dimension]. Baltiyskiy region [The Baltic Region], 2016, vol. 8, no. 2, pp. 30-47.

Krivonozhko V.E., Lychev A.V. Analiz deyatelnosti slozhnykh sotsialno-ekonomicheskikh sistem [Analysis of the Activities of Complex SocioEconomic Systems]. Moscow, Maks Press Publ., 2010. 208 p.

Larionov I.K. Mezoekonomika: ucheb. posobie [Mesoeconomics. Study Guide]. Moscow, Dashkov i K Publ., 2001. 444 p.

Mesarovich M., Mako D., Takahara I. Teoriya ierarkhicheskikh mnogourovnevykh system [Theory of Hierarchical Multilevel Systems]. Moscow, Mir Publ., 1973. 344 p.

Nogin V.D. Prinyatie resheniy v mnogokriterialnoy srede: kolichestvennyy podkhod [Decision Making in a Multi-Criteria Environment: Quantitative Approach]. Moscow, FIZMATLIT Publ., 2002. 144 p.
Programma dlya EVM «Programmnyy kompleks dlya otsenki funktsionirovaniya slozhnykh sistem $i$ prinyatiya resheniy "EFRA" ("EFRA")》. Svidetelstvo Federalnoy sluzhby po intellektualnoy sobstvennosti, patentam $i$ torgovym znakam ob ofitsialnoy registratsii programmy dlya EVM № 220614151 ot 26.03.2020. [Computer Program "Software Package for Evaluating the Functioning of Complex Systems and Making Decisions "EFRA". Certificate of the Federal Service for Intellectual Property, Patents and Trademarks on the Official Registration of Computer Programs No. 220614151 of March 26, 2020)].

Morozova T.G. et al. Regionalnaya ekonomika: uchebnik [Regional Economics: Textbook]. Moscow, UNITY-DANA Publ., 2012. 527 p.

Saati T. Prinyatie resheniy: metod analiza ierarkhiy [Decision Making: Hierarchy Analysis Method]. Moscow, Radio i svyaz Publ., 1993. 278 p.

Sreda razrabotki Mathlab [Matlab Development Environment]. URL: https://www.mathworks. com/products/matlab.html. (accessed 4 May2020).

Federalnyy zakon Rossiyskoy Federatsii ot 21.07.1993 № 5485-1 «O gosudarstvennoy tayne» [Federal Law of the Russian Federation No. 5485-1 of July 21, 1993 “'On State Secret”]. Access from Reference Legal System 'KonsultantPlyus'.

Federalnyy zakon Rossiyskoy Federatsii ot 29.07.2004 № 98-FZ «O kommercheskoy tayne» [Federal Law of the Russian Federation No. 98-FZ of July 29, 2004 "On Trade Secret"]. Access from Reference Legal System 'KonsultantPlyus'.

Federalnyy zakon Rossiyskoy Federatsii ot 27.07.2006 № 149-FZ «Ob informatsii, informatsionnykh tekhnologiyakh i o zashchite informatsii» [Federal Law of the Russian Federation No. 149-FZ of July 27, 2006 “About Information, Information Technologies and Information Protection"]. Access from Reference Legal System 'KonsultantPlyus'.

Zhukov R.A., Polyakov V.A., Vasina M.V., Soboleva D.V. Formirovanie sistemy vzglyadov na protsess upravleniya ustoychivym razvitiem regiona [The Formation of a System of Views on the Process of Management of Sustainable Development of the Region]. Nauchnye issledovaniya i razrabotki. Ekonomika [Scientific Research and Development. Economics], 2018, vol. 6, no. 6, pp. 48-53.

Bobylev S.N., Grigoryeva L.M. Chelovek i innovatsii: Doklad o chelovecheskom razvitii v Rossiyskoy Federatsii za 2018 god [Human and Innovation. Human Development Report in the Russian Federation-2018]. Moscow, Analiticheskiy tsentr pri Pravitelstve RF, 2018. 172 p. 
Chernitsky S.V. Kompleksnaya metodika otsenki ekonomicheskogo potentsiala regionov [Integrated Methodology for Assessing the Economic Potential of Regions]. Istoricheskaya i sotsialnoobrazovatelnaya mysl [Historical and SocialEducational Idea], 2014, no. 5 (27), pp. 208-213.

Bertalanffy L. General System Theory - A Critical Review. General Systems, 1962, vol. 7, pp. 1-20.

Cobb C. W., Douglas P.H. A Theory of Production. American Economic Review, 1928, no. 18, pp. 139-165.
Corbo V., Meller P. The Translog Production Function. Some Evidence from Establishment Data. Journal of Econometrics, 1979, no. 10, pp. 193-199.

Charnes A., Cooper W.W., Huang Z.M., Sun D.B. Polyhedral Cone-Ratio DEA Models with an Illustrative Application to Large Commercial Banks. Journal of Econometrics, 1990, vol. 46, no. 1-2, pp. 73-91.

Solow R.M. A Contribution to the Theory of Economic Growth. The Quarterly Journal of Economics, 1956, vol. 70, no.1, pp. 65-94.

\section{Information About the Author}

Roman A. Zhukov, Candidate of Sciences (Physics and Mathematics), Associate Professor, Department of Mathematics and Informatics, Financial University Under the Government of the Russian Federation (Tula Branch), Oruzheynaya St, 1a, 300012 Tula, Russian Federation, pluszh@mail.ru, https://orcid.org/0000-0002-2280-307X

\section{Информация об авторе}

Роман Александрович Жуков, кандидат физико-математических наук, доцент кафедры математики и информатики, Тульский филиал Финансового университета при Правительстве РФ, ул. Оружейная, 1a, 300012 г. Тула, Российская Федерация, pluszh@mail.ru, https://orcid.org/0000-0002-2280-307X 